\title{
Uncooled Detectors of Continuum Terahertz Radiation
}

\author{
P. Kaufmann \\ Universidade Presbiteriana Mackenzie, CRAAM - Escola de Engenharia, São Paulo, SP, Brazil, and \\ Universidade Estadual de Campinas, CCS - Centro de Componentes Semicondutores, Campinas, SP, Brazil, \\ pierrekau@gmail.com \\ R. Marcon \\ Universidade Estadual de Campinas, IFGW, Campinas, SP, Brazil and Observatório Solar "Bernard Lyot", \\ Campinas, SP, Brazil, rmarcon@mpcnet.com.br \\ A.S. Kudaka, M. M. Cassiano, L.O.T. Fernandes \\ Universidade Presbiteriana Mackenzie, CRAAM - Escola de Engenharia, São Paulo, SP, Brazil, \\ kudaka@mackenzie.br \\ A. Marun, P. Pereyra, R. Godoy \\ Complejo Astronomico El Leoncito, CONICET San Juan, Argentina, amarun@casleo.gov \\ E. Bortolucci, M. Beny Zakia, J.A. Diniz, A.M. Pereira Alves da Silva \\ Universidade Estadual de Campinas, CCS - Centro de Componentes Semicondutores, Campinas, SP, Brazil, \\ emilio@ccs.unicamp.br \\ A.V. Timofeevsky, V.A. Nikolaev \\ Tydex J.S. Co, St. Petersburg, Russia, alexandertymofeevsky@tydex.ru
}

\begin{abstract}
THz continuum spectral photometry has new and unique applications in different civil and military areas presenting a number of distinctive advantages on the well known microwaves or mid- to near-infrared technologies. $\mathrm{THz}$ sensing is essential to investigate the emission mechanisms by high energy particle acceleration processes. Technical challenges appear to diagnose radiation produced by solar flare burst emissions measured from space as well as radiation produced by high energy electrons in laboratory accelerators. THz filters and detectors have been investigated for the construction of solar flare high cadence radiometers to operate outside the terrestrial atmosphere. Experimental setups have been assembled for testing $\mathrm{THz}$ continuum radiation response from distinct detectors: adapted commercial microbolometer array, pyroelectric module, and optoacoustic (Golay cell). The results permitted the final design of a $\mathrm{THz}$ double radiometer using Golay cells to be flown in stratosphere balloon missions.
\end{abstract}

Index Terms - Far IR continuum spectral photometry, THz radiometers, THz sensors, Solar THz radiation

\section{INTRODUCTION}

Technologies for photometry and imaging in the THz range (arbitrarily $0.1-30 \mathrm{THz}$ ) are in full expansion for a variety of new and unique applications in different civil and military areas presenting a number of distinctive advantages on the well known microwaves or mid- to near-infrared technologies. THz radiation propagates well through cloth, dust and fog [1,2,3]. Sensing in this range 
is proving to be particularly useful to determine internal characteristics of materials, in the search for drugs, mines, and explosive materials. New biological and medical $\mathrm{THz}$ imaging applications are far reaching. Aerospace $\mathrm{THz}$ remote sensing applications include new approaches to determine atmospheric inhomogeneities and cloud characteristics $[4,5,6]$.

Photometry and imaging at $\mathrm{THz}$ frequencies have important application in the diagnostics of radiation produced by high energy electrons, observed in laboratory accelerators [7] as well as by thermal and non-thermal space plasmas [8,9]. Solar flare accelerates electrons to high energies. Their radiation by synchrotron mechanism predicts intense fluxes in the far IR or THz range of frequencies [10]. The radiometry of temperature enhancements above a pre-existent bright level - as it is the case of flare radiation excess over the solar disk intense emission - requires the effective suppression of the incoming visible and near-infrared (NIR) radiation. This has been accomplished with the use of a number of THz low-pass filters [11], consisting in a combination of rough surface mirrors $[12,13,14]$ and commercially available membranes $[15,16]$. We present the performance of distinct uncooled sensors in response to black body $\mathrm{THz}$ radiation for different sensors: microbolometer array, pyroelectric module, and Golay cell.

\section{TEST OF UNCOOLED THZ DETECTORS}

\section{A. Adapted microbolometer array}

A custom-made detector consisted in a room-temperature vanadium oxide micro-bolometer focal plane array (FPA) camera IRM 160A with HRFZ-Si THz window provided by INO Company, Quebec, Canada [17]. The camera total-power response for black body temperatures ranging 300$1000 \mathrm{~K}$ was measured at El Leoncito laboratory. A nichrome resistor, assumed as close to an ideal black body radiator, was placed at the focus of $150 \mathrm{~mm}$ concave reflector to produce an image occupying nearly $70 \%$ of the FPA. We selected the Region Of Interest (ROI) over the area in the frame filled by the heated resistor image. All pixels readings on the ROI were added and averaged for every frame reading, quoted in camera reading units. Several sets of measurements were taken, for temperatures ranging from ambient (about $290 \mathrm{~K}$ ) to about $900 \mathrm{~K}$, without any low-pass filter, and using the two membrane low-pass filters described elsewhere $[11,15,16]$. One set of measurements is summarized in Fig. 1 (a). Figure 1(b) shows another expanded set of data showing the camera response using the Zitex G110 [15] and the TydexBlack [16] low-pass membranes.

The fluctuation of data points can be attributed to measurement uncertainties (of about \pm 1 reading unit), since they were taken with high cadence (30 frames/s). It can be noted that the camera readings with Zitex G110 low pass filter interposed is about 20-40 reading units above the TydexBlack readings, for the whole range of temperatures. This effect was repeatedly observed for all series of measurements. It might correspond to the fraction of power in the visible-NIR transmitted by Zitex G110 [11]. 


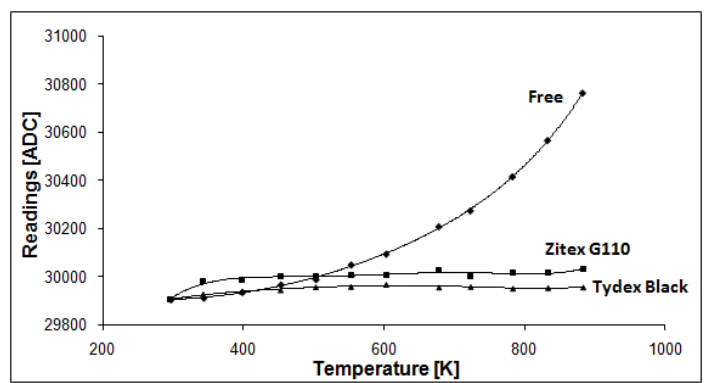

(a)

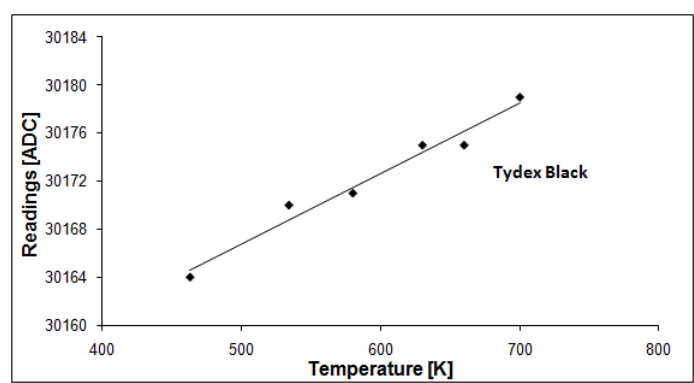

(b)

Fig. 1 - The response of INO IRM 160A camera FPA with HRFZ-Si window to black body radiation: (a) for the whole spectrum and through the low-pass membranes filters for the THz spectral contribution. (b) Independent expanded plot for TydexBlack low-pass filter. The output ordinates are in ADC camera reading units.

The substantial reduction in the camera response to black body temperature changes when interposing the low-pass membranes proves their effectiveness in the readings increase suppressing the visible and NIR radiation. Indeed the predicted ratio of power increase for a black body heated about $100 \mathrm{~K}$, at the $700 \mathrm{~K}$ level, for the whole main spectrum $(\lambda>0.5 \mu \mathrm{m})$ in comparison to the $\mathrm{THz}$ part of the spectrum $(\lambda>15 \mu \mathrm{m})$ is close to 60 . This might be compared to ratio of about 40 between the camera ROI readings increase in that range compared to with the membrane low-pass filters.

The camera scale of about $25 \mathrm{~K}$ per reading unit ( \pm 1 reading unit) was too large to allow any measurable differences when adding one resonant metal mesh band-pass filter [18].

\section{B. Pyroelectric detector module}

The pyroelectric modular detector made by Spectrum Detector Inc. [19], model SPH65-THz, was tested at the laboratory of the Center for Semiconductor Components (CCS), State University of Campinas. The setup utilised a standard laboratory Newport model 67030 black body source with a build-in wheel chopper, set at $20 \mathrm{~Hz}$. The detector response to black body temperatures is shown in Fig. 2 for open conditions (responding to the visible - THz range) and for low-pass membranes interposed.

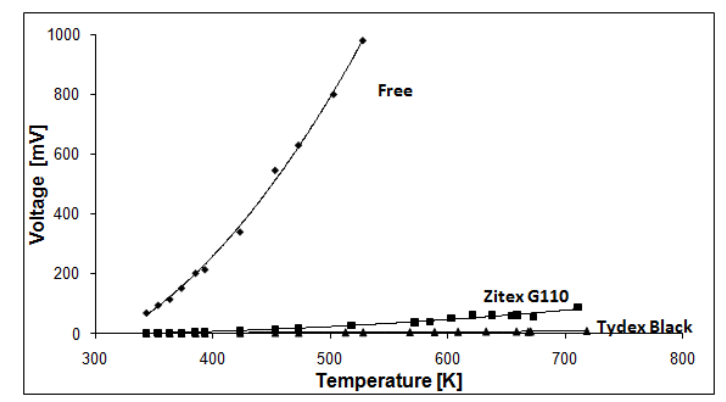

Fig. 2 - The Spectrum Detector SPH65-THz pyroelectric module peak-to-peak mV response to black body temperatures for open conditions and with low-pass membrane filters interposed: Zitex G110 and TydexBlack, which are expanded in Fig. 3. 
The pyroelectric sensor response to blackbody temperature variations was considerably better defined, compared to the microbolometer array response. The detector readings with Zitex G110 lowpass filter are larger than the readings with TydexBlack, increasing with temperatures (see left panel in Fig. 3). This effect might correspond to the fraction of power in the visible-NIR transmitted by Zitex G110 [11]. The small proportion of power allowed through with the use of the low-pass filter is consistent with the efficient suppression of visible and NIR radiation. Transmission tests were performed by adding a $2 \mathrm{THz}$ metal mesh band-pass filter [18]. For higher temperatures there were indications on power reduction, qualitatively consistent with the expected band-pass. The measured output, however, was close to the output reading fluctuations, preventing the reliable reading of several data points, for different temperatures.
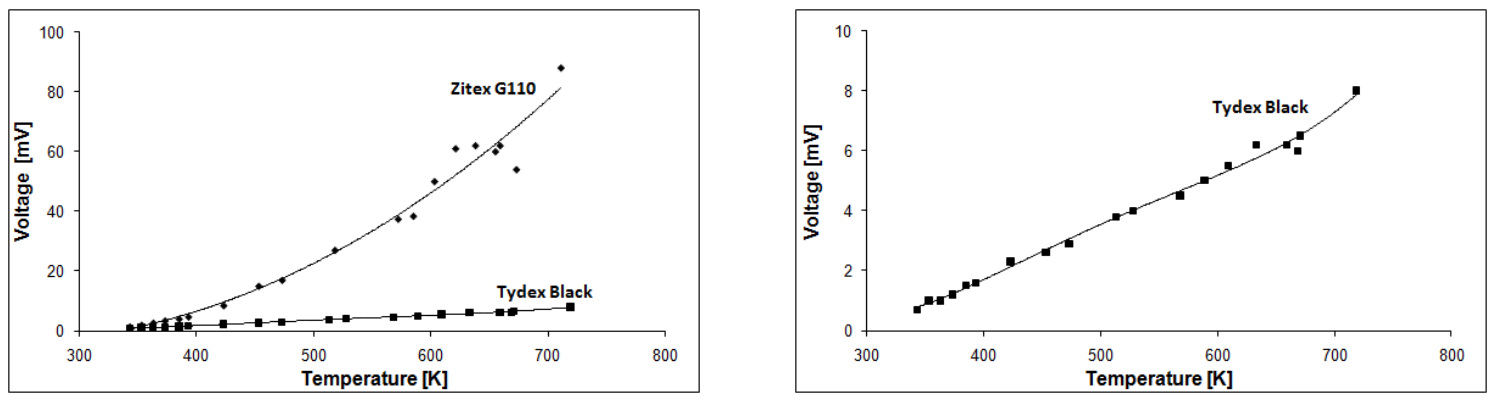

Fig. 3 - Expanded scale peak-to-peak mV response for the Spectrum Detector SPH65-THz pyroelectric module to black body temperatures with low-pass membrane filters interposed: Zitex G110 and TydexBlack.

\section{The opto-acoustic detector (Golay cell)}

The performance of the Tydex model GC-1P Golay cell sensor [20] was investigated on similar conditions. The first set of measurements was carried out at Tydex Company, using a standard black body, $2 \mathrm{~mm}$ diameter diaphragm in front of the extended black body source and a wheel chopper. Filters and diaphragm were interposed in front of the Golay cell. We report here the measurements taken with the diaphragm close to the detector, chopper frequency of $20 \mathrm{~Hz}$.

A 2 THz band-pass resonant metal mesh filter, fabricated separately [18], was added to the lowpass membrane filter, producing the nearly linear response shown in Fig. 4. The reduction in detector outputs for low-pass filter (frequencies $<15 \mathrm{THz}$ ) and for band-pass filter added $(2 \pm 0.2 \mathrm{THz})$ to the low-pass filter are consistent with the spectral power reduction in the respective band. The sensitivity of the Golay cell can be further increased with the use of the input photon collecting cone and/or by adding a small reflecting aperture. 


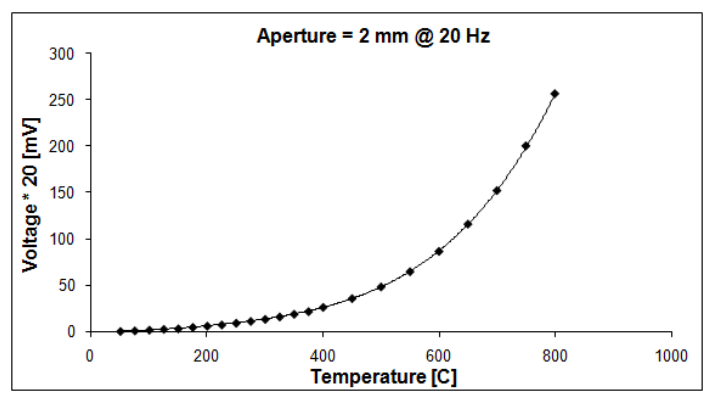

(a)

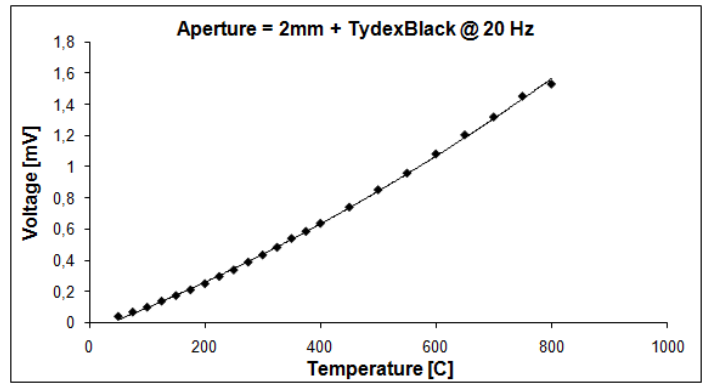

(b)

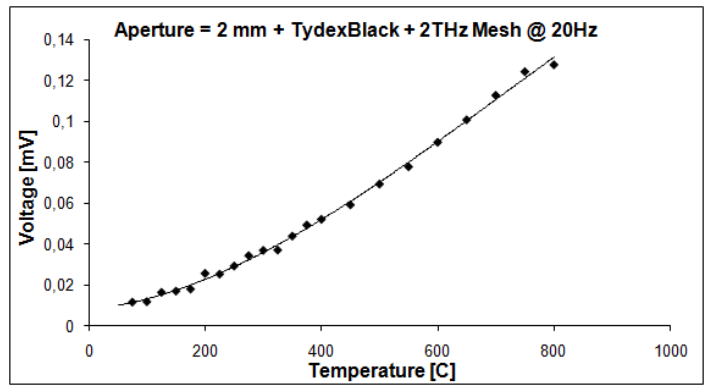

(c)

Fig. 4 - The Tydex GC-1P opto-acoustic detector (Golay cell) response to black body temperature variations through a $2 \mathrm{~mm}$ aperture. (a) No filter interposed. (b) TydexBlack low-pass filter interposed and (c) with low-pass filter TydexBlack and 2

THz metal mesh band-pass filter interposed.

\section{CONCLUDING REMARKS}

The detection of black body $\mathrm{THz}$ radiation has been tested for microbolometer focal plane array, pyroelectric module, and opto-acoustic detector (Golay cell). The Golay cell exhibited superior sensitivity for the detection of weak power fraction enhancement as a function of temperature variations as measured with a band-pass of $\pm 10 \%$ centered at the THz frequency. The results obtained in this study provided the necessary parameters needed for the design of a $\mathrm{THz}$ radiometer intended for solar flare observations from a space platform.

A double THz solar radiometer system is currently being developed to operate at center frequencies of 3 and $7 \mathrm{THz}$, which concept design is shown in Figure 5. It was provisionally named SOLAR-T 
[21]. The Golay cell sensor is preceded by a tuning fork resonant chopper. The incoming solar signal passes by a photon concentrator consisting in a Cassegrain aperture optics that forms the solar disk image with a linear size smaller than the Golay cell sensitive sensor size (i.e., smaller than about 5 $\mathrm{mm})$. The reflectors' surfaces are roughened [14] in order to diffuse most of the visible and near IR thermal radiation (i.e. for $\lambda \leq 20 \mu$ ). The windows at the vertices of the reflectors consist of a sandwich of a plate with good THz transmission (such as TPX), a low pass membrane filter $(\lambda \geq 20 \mu)$ made of Zitex G110 and TydexBlack membranes [11] and resonant metal mesh band-pass filters [18] with center frequencies at 3 and $7 \mathrm{THz}( \pm 10 \%$ bandwidth) .
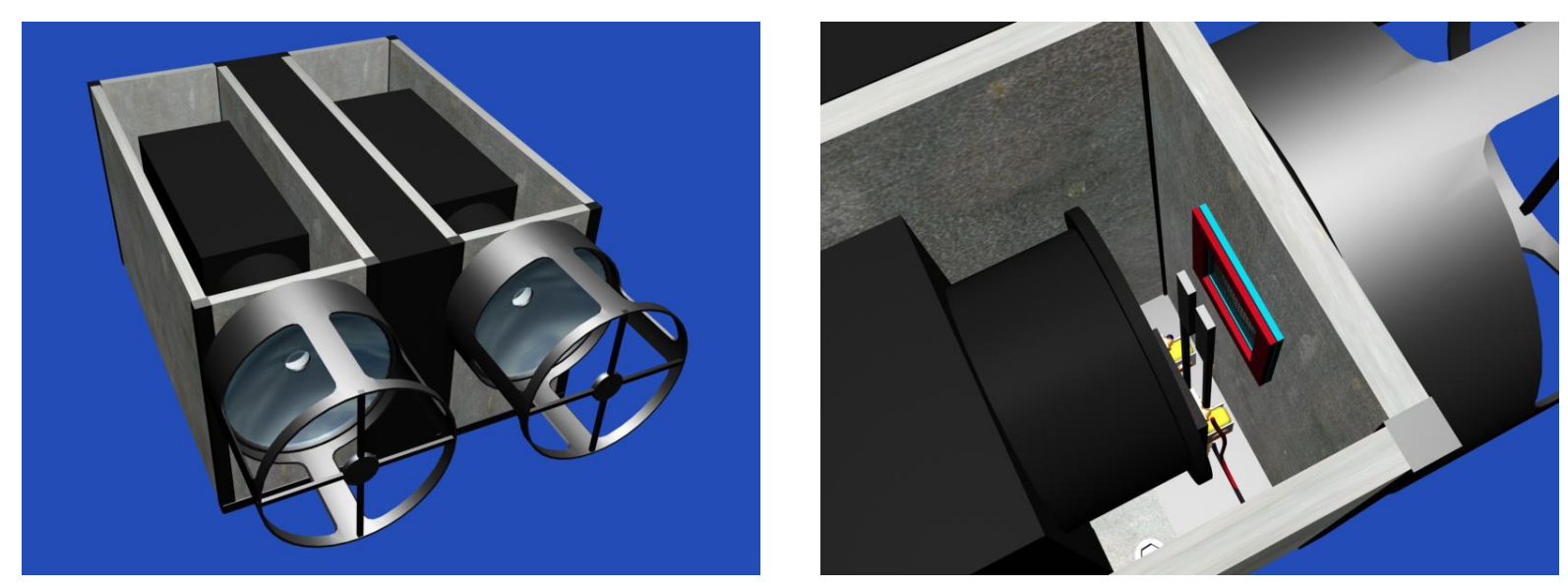

Fig. 5 - Concept design of the double 3 and $7 \mathrm{THz}$ solar radiometer system, provisionally called SOLAR-T [21] to be flown in three stratosphere balloon long duration flights over USA, Antarctica and Russia. The left panel shows the two

Cassegrain telescopes ( $70 \mathrm{~mm}$ in diameter) with roughened primary mirrors. The two Golay cells and electronics box are seen from the top. The right panel shown the input filters sandwich made of the band-pass resonant metal mesh filter (centered at 3 and $7 \mathrm{THz}$ ) plus the low-pass membrane filter plus the THz TPX window [21]

The THz radiometers' SOLAR-T system is planned to be flown in long duration flights over Russia (about one week) sometime between 2013-2016[21], in a cooperation with Moscow Lebedev Physics Institute, and on USA and Antarctica (about two weeks circumnavigation), in 2012 and 2013, respectively, in cooperation with University of California, Berkeley, together with their GRIPS gamma-ray experiment [22].

\section{ACKNOWLEDGMENTS}

This research was partially supported by Brazilian agencies FAPESP, CNPq, CNPq-FAPESP/INCTNAMITEC, Mackpesquisa and Argentina agency CONICET.

\section{REFERENCES}

[1] Harris, D.C., "Materials for infrared windows and domes", SPIE Optical Engineering Press, Washington, USA,1999.

[2] Siegel, P.H., "THz Technology: An Overview", International Journal of High Speed Electronics and Systems, 13, n², 2003, 1-44.

[3] Mlynczak., M., Johnson, D., Bingham, D.G. et al., "Far-Infrared Spectroscopy of the Troposphere (FIRST) project", Proceedings of Geoscience and Remote Sensing Symposium, 1, 2003, 512. 
[4] Sherwin M. S., Schmuttenmaer C. A., Bucksbaum P. H., Proceedings of DOE-NSF-NIH Workshop on Opportunities in THz Science, Arlington, VA, 2004.

[5] Kinch, M.A., "Infrared detector materials", SPIE Optical Engineering Press, TT76, Washington, USA, DC, 2007.

[6] Strabala K. I., Ackerman S. A. and Menzel W. P., "Cloud Properties inferred from 8-12- $\mu$ m Data", Journal of Applied Meteorology, 33, 1994, 212.

[7] Williams, G. P., "FAR-IR/THz radiation from the Jefferson Laboratory, energy recovered linac, free electron laser", Rev. Sci. Instrum. 73, 2002, 1461-1463.

[8] Kaufmann, P. and Raulin, J.-P., "Can microbunch instability on solar flare accelerated electron beams account for bright broadband coherent synchrotron microwaves?", Phys. Plasmas 13, 2006, 070701-070701-4.

[9] Klopf, J. M., in 1st SMESE Workshop, 10-12 March, Paris, France, 2008.

[10] Kaufmann, P. et al., Astrophys. J., 603, 2004, L121.

[11] Kaufmann, P. et al., "Continuum Terahertz Radiation Detection Using Membrane Filters", International Microwave and Optoelectronics Conference, SBMO \& IEEE-MTT, Nov. 3-6 2009, Belem, Pará, Brazil, 2009, 262-266.

[12] Bennett, H.E. and Porteus, J. O. "Relation between surface roughness and specular reflectance at normal incidence", $J$. Opt. Soc. Am. 51, 1961, 123-129.

[13] Kostiuk.T. and Deming, D., "A solar infrared photometer for space flight application”, Infrared Physics 32, 1991, 225233.

[14] Kornberg, M. et al., "Rough Mirrors for the THz Frequency Range", Proc. MOMAG 2008 - 13th SBMO and 8th CBMAG, Florianópolis, SC, Brazil, 7-10 September, 2008, 365-367.

[15] Benford, D.J., Gaidis, M.C., and Kooi, J.W., "Optical properties of Zitex in the infrared to submillimeter", Applied Optics 42, 2003, 5118-5122.

[16] www.tydex.ru, Tydex JSCo, St. Petersburg, Russia, Technical Note on THz materials and components, 2008.

[17] http://www.ino.ca/en-CA

[18] Melo, A.M. et al., "Metal mesh resonant filters for terahertz frequencies", Applied Optics 47,2008, 6064-6069.

[19] http://www.spectrumdetector.com, 2009.

[20] http://www.tydexoptics.com/products1/thz_optics/golay_cell/, 2009.

[21] Kaufmann, P. et al., "Search for Continuum Solar Flare Radiation in the Terahertz Range", in Proc. of $I R M M W-T H z$ 2010 Conference, Rome, Italy, 5-10 September, 2010.

[22] Shih, A. et al., The Gamma-Ray Imager/Polarimeter for Solar Flares (GRIPS), American Geophysical Union, Fall Meeting, abstract SM11B-1602,2008. 